\title{
Coronal quasi-periodic fast-propagating magnetosonic waves observed by SDO/AIA
}

\author{
Yuandeng Shen \\ Yunnan Observatories, Chinese Academy of Sciences, Kunming 650216, China \\ email: ydshen@ynao.ac.cn
}

\begin{abstract}
Coronal quasi-periodic fast-propagating (QFP) magnetosonic waves are scare in previous studies due to the relative low temporal and spatial resolution of past telescopes. Recently, they are detected by the Atmospheric Imaging Assembly (AIA) on board the Solar Dynamics Observatory (SDO). Here, two cases of QFP waves are presented. The analysis results indicate that QFP waves are tightly associated with the associated flares. It is indicate that QFP waves and the associated flares are possibly driven by the same physic process such as quasiperiodic magnetic reconnection process in producing flares.
\end{abstract}

Keywords. Waves, Flares, Magnetic fields, Oscillations, Atmosphere

\section{Introduction}

The observations of coronal QFP waves are usually imaged by the SDO/AIA $171 \AA$ channel. They are always associated with flares, and have multiple arc-shaped wavefronts that emanate successively from the flare kernel and propagate outward along coronal loops. Their speed can range from several hundred to a few thousand $\mathrm{km} \mathrm{s}^{-1}$, and the periods are of tens of seconds to a few minutes. The first QFP wave was reported by Liu et al. 2011. From then on, the QFP wave has attracted a lot of attention due to the possible application in coronal seismology and the role played in the coronal heating problem. So far, more observations have been documented in (e.g., Shen \& Liu 2012, Liu et al. 2012, Shen et al. 2013, Yuan et al. 2013, Nisticò et al. 2014, Kumar \& Innes 2015). In the meantime, simulation experiments have also been performed to understand the driving mechanism and propagation properties of QFP waves (Ofman et al. 2011, Pascoe et al., 2013, 2014, Yang et al. 2015). Here, two QFP wave events are analyzed to provide some new clues for understanding the driven mechanism and propagation properties of QFP waves.

\section{Results}

The first QFP wave case occurred on May 30, 2011, which accompanied by a C2.8 flare and imaged by the SDO/AIA $171 \AA$ channel. The wave trains emanated from the flare kernel successively and propagated along a group of open coronal loop, and the wavefronts could be divided into three sub QFP waves (see arrows in Figure 1. (b)-(c)), which have difference periods, amplitude, and phase speeds, but they are all related to impulsive radio bursts (Yuan et al. 2013). A $k-\omega$ diagram is generated over the wavefront region, which reveals that there are many frequencies in the wave (see Figure 1. (e)). By analyzing the frequencies of the associated flare, it is found that the flare's frequencies are all included in the QFP wave's frequency spectrum. This suggests that the flare and the QFP waves were possibly excited by a common physical process. On the other hand, a few low frequencies (e.g., 2.5 and $0.7 \mathrm{mHz}$ ) revealed by the $k-\omega$ diagram cannot be found in the accompanying flare. This is possibly due to the leakage of the pressuredriven oscillations from the photosphere into the low corona, which should be a noticeable 


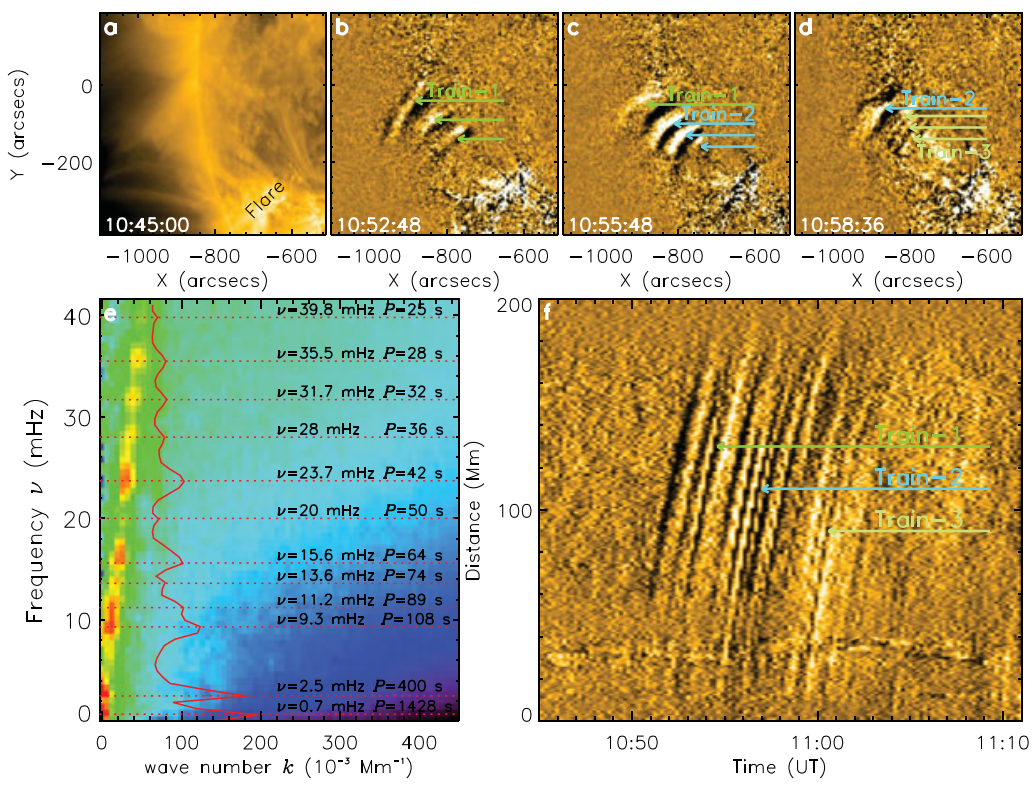

Figure 1. The QFP wave on May 30, 2011. a, direct $171 \AA$ image. b - d, running difference $171 \AA$ images with arrows indicating the wave trains. e, Fourier power $(k-\omega)$ of the 171 Årunning difference images during 10:48-11:00 UT over the wavefront region. $f$, time-distance plot obtained from $171 \AA$ running difference images along a line perpendicular to the wavefronts, and arrows indicating the propagating wave trains.

mechanism for driving QFP waves in corona. The time-distance plot shows that the phase speeds of three sub QFP waves are 735, 845, and $820 \mathrm{~km} \mathrm{~s}^{-1}$, and their decelerations are $1.35,2.27$, and $1.31 \mathrm{~km} \mathrm{~s}^{-2}$, respectively.

The other QFP wave occurred on April 23, 2012, which accompanied by a C2.0 flare and propagated along a loop system (see Figure 2. (a)-(c)). A flare ribbon near the guiding loop's footpoint may directly lead to the QFP wave. The wave trains are firstly observed in AIA $171 \AA$ images, after the interaction with a perpendicular loops on the path, they suddenly appeared in the $193 \AA$ channel (see Figure 2. d-e). The average phase speeds before and after the interaction are about 689 and $343 \mathrm{~km} \mathrm{~s}^{-1}$ in the $171 \AA$, and it is about $362 \mathrm{~km} \mathrm{~s}^{-1}$ in the $193 \AA$. Periodic analysis indicate that the periods of the QFP wave before and after the interaction are all of $80 \mathrm{~s}$ in the $171 \AA$ observations, and that measured from the $193 \AA$ images are also the same. Interestingly, the period of the accompanying flare is the same with the wave trains, suggesting that the QFP wave and the flare are all the result of a common physical process.

\section{Conclusions and Discussions}

Based on the two cases of QFP waves, one main common characteristic is that QFP waves have similar periods with the associated flares. This indicate that QFP waves and flares are the two sides of manifestations of a single physical process. Analysis results indicate that the periodic releasing of energy bursts through some nonlinear processes in the magnetic reconnection process, which produces flares, could be a possible explanation.

In the second event, the sudden deceleration of the wave trains in $171 \AA$ images and their appearance in $193 \AA$ observations could be interpreted through a geometric effect and the density increase of the guiding loop system, respectively. It is well known that the distribution of magnetic fields is very complex, but the basic configuration should 

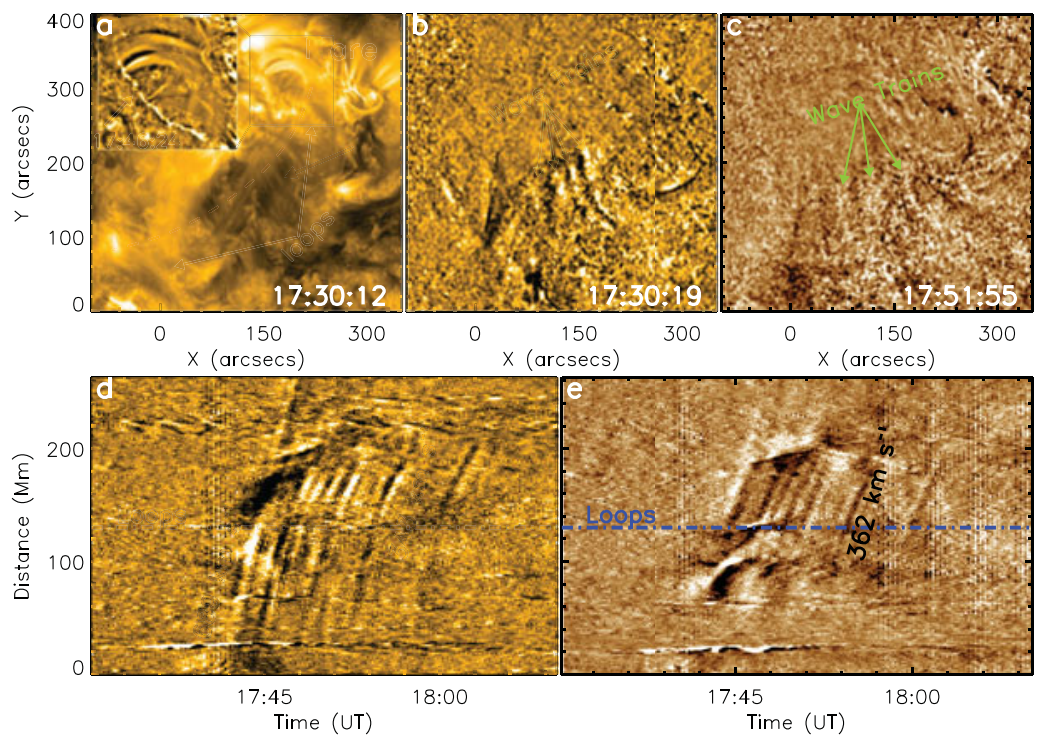

Figure 2. The QFP wave on April 23, 2012. a, direct $171 \AA$ image. b and c, $171 \AA$ and $193 \AA$ running difference images, respectively. $\mathrm{d}$ and e, time-distance plots made from $171 \AA$ and 193 A running difference images along the dashed curve showing in panel a. The inset in panel a shows the detail of the flare region, and the blue dashed lines in panels $\mathrm{d}$ and e indicate the position of the perpendicular loop.

be an upward funnel-like shape. Here, the guiding loops may change their inclination angle significantly when approaching the perpendicular loop system, and thus become more curved upwardly. Therefore, due to the projection effect the observed wave speed can decrease to a small value within a short timescale. On the other hand, when the wave-guiding loops interact with the perpendicular loops, the wave trains will cause a strong compression of the guiding fields, which would increase the density of the guiding loops quickly and thereby decrease the speed of the wave trains within a short timescale. In addition, the compression can still cause a possible adiabatic heating that dissipates the wave energy and thus result in the wave trains in the $193 \AA$ observations.

\section{Acknowledgements}

This work is supported by Chinese foundations (11403097, 2015FB191), and the Youth Innovation Promotion Association (2014047).

\section{References}

Kumar, P. \& Innes, D. E. 2015, ApJ, 803, 23

Liu, W., Title, A. M., Zhao, J. W., Ofman, L., \& Schrijver, C. J., et al. 2011, ApJL, 736, L13

Liu, W., Ofman, L., Nitta, N. V., Aschwanden, M. J., \& Schrijver, C. J., et al. 2012, ApJ, 753, 52

Nisticò, G., Pascoe, D. J., \& Nakariakov, V. M. 2014, A\&A, 569, A12

Ofman, L., Liu, W., Title, A., \& Aschwanden, M. 2011, ApJL, 740, 33

Pascoe, D. J., Nakariakov, V. M., \& Kupriyanova, E. G. 2013, A\&A, 560, 97

Pascoe, D. J., Nakariakov, V. M., \& Kupriyanova, E. G. 2014, A\&A, 568, A20

Shen, Y. \& Liu, Y. 2012, ApJ, 753, 53

Shen, Y., Liu, Y., Su, J. T., Li, H., \& Zhang, X. F., et al. 2013, SoPh, 288, 585

Yang, L., Zhang, L., He, J., Peter, H., Tu, C., et al. 2015, ApJ, 800, 111

Yuan, D., Shen, Y., Liu, Y., Nakariakov, V. M., \& Tan, B., et al. 2013, A\&\&A, 554, A144 